\title{
The man who swallows forks - a complex case of repetitive ingestion of foreign bodies
}

$\underline{\text { R. Gasparinho }}^{1}$, L. P. Ferreira ${ }^{1}$, A. R. Marques ${ }^{1}$, N. A. Fernandes ${ }^{1}$, N. Santos ${ }^{1}$, M. Martins ${ }^{1}$, A. Alho $^{1}$, A. Rosa .

1- Department of Psychiatry and Mental Health, Hospital Distrital de Santarém, Portugal

\section{Background}

Intentional ingestions of foreign bodies (IIFB) is a rare clinical problem, mostly seen in psychiatric disorders, prison inmates and mental impairment ${ }^{(1-4)}$. IIFB differential diagnosis includes factitious disorder, pica, malingering, psychotic disorders, mood disorders, obsessive-compulsive disorder, post-traumatic stress disorder, personality disorders, impulse control disorders, alcohol and substances abuse $(1, \underline{3})$. Among these patients the most commonly reported disorder is malingering, typically reported in prison inmates and institutionalized psychiatric patients

When examining IIFB it is important to keep in mind that not every case of IIFB is associated with an underlying psychiatric disorder (4).

Our objective is to report a case of repetitive intentional ingestion of foreign bodies.

\section{Methods}

Inpatient clinical assessment for 25 days, follow-up outpatient clinical assessment for 24 months and clinical file consultation. Pubmed search with MeSH terms: "Self-Injurious Behavior" AND "Foreign Bodies". Pubmed search with key terms: "Ingestion"; "Incarceration"; "Prison". Search was restricted to articles written in English in the last 15 years.

\section{Case Report}

We present the case of a 41-year-old male, single, currently living with his parents. He has a history of childhood trauma (saw his brother dying at the age of 6 ). He has no known family psychiatric history. He was incarcerated for 17 years (released at the age of 39). While incarcerated he repeatedly displayed non-suicidal self-harm and suicidal behavior, and intentionally ingested foreign bodies 35 times (including a $15 \mathrm{~cm}$ metal object, plastic and metal forks, razor blades, nail clippers).

Since his release from prison 26 months ago, alcohol and cannabis use disorders became evident and he was admitted in a therapeutic community soon after (where he stayed only 3 months). Relapses in substance abuse have been frequent during our follow-up. He kept displaying the same behavior and now has more than 41 intentional ingestions of foreign bodies (which resulted in 4 hospitalizations in the Surgery ward and 2 in the Psychiatry ward (examples in figures 1, 2, 3 and 4). He has $\mathbf{3 2}$ attendances to the emergency department, was submitted to 5 laparotomies, ingested razor blades and forks (some ingestions occurred while hospitalized). When questioned why he would display this behavior he often replied that he did it without thinking, that there was no reason, that he did it because he was "bored" or because his parent's haven't given him money, that he felt lonely or irritated, and sometimes he didn't justified his behavior. No pharmacological approach was proven effective in reducing IIFB behaviors.

Most of his IIFB were treated conservatively (watchful waiting and several endoscopies) and did not require long periods of hospitalization. It's also clear that most of his IIFB had an impulsive nature, and that he exhibits traits of poor frustration tolerance, dependence and attention-seeking behavior

Nowadays, consequence of his repeated IIFB which resulted in several surgical procedures he has an abdominal hernia and gastroesophageal reflux disease. At the moment he remains an outpatient and voluntarily accepts appointments and pharmacological treatment (selective serotonin reuptake inhibitor and mood stabilizer)

\section{Discussion}

Through the years our patient has been diagnosed with several different disorders, being the most frequent alcohol and cannabis use disorders, personality disorder, depressive disorder, malingering, impulsive control disorder and light mental impairment. This diagnostic instability reflects the complexity of the case.

To this day, despite different treatments and a multidisciplinary approach we still haven't been able to eradicate this destructive behavior and successfully prevent episodes of IIFB. There is no specific treatment for IIFB. In this case psychopharmacological treatment has been symptomatic and focused in reducing impulsivity.

There is few literature regarding successful prevention strategies. Control of objects in patient's surrounding seems to be effective in prevention of other types of self-harm but it is virtually impossible in the case of objects which may be potentially ingested Nevertheless prevention strategies should include decreasing access to ingestible objects and increasing psychotherapy Pharmacological treatment should be tailored to the patient's specific psychiatric diagnosis ${ }^{(5)}$. Patients should return to their usual environment as soon as feasible to avoid secondary gain and to reduce hospitalization associated costs. Also, the avoidance of unnecessary endoscopic interventions may help prevent repeat IIFB

\section{Conclusion}

IIFB represents a healthcare problem which carries a heavy burden to patients, their families and to healthcare staffs' and system. There are many challenges in dealing with patients who display repetitive IIFB behaviors: it carries a sense of insidiousness in the lack of outward evidence that harm has been done and in the risk of latent further injury (6), it may originate countertransference and diminish the morale of their care providers $(2, \underline{6}, \underline{7})$. This case aims to illustrate the difficulties in preventing IIEF and the clinical, legal and ethical implications when dealing with this behavior.
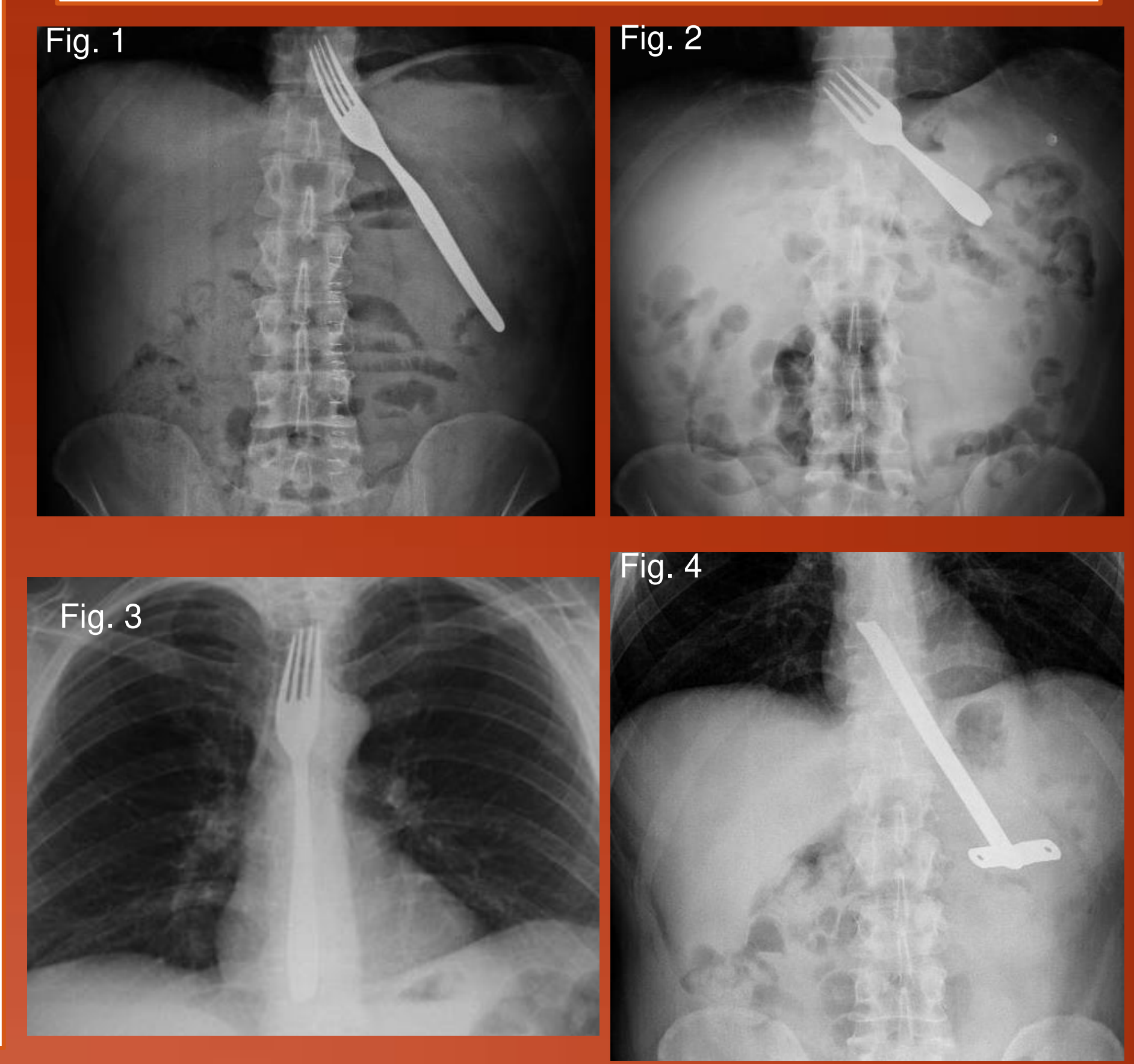

\section{Bibliography}

1. Galczynski A, Cieplinska E, Konturek A. Habitual Intentional Foreign Body Ingestion - A Literature Review. Polski przeglad chirurgiczny. 2016;88(5):290-7. 2. Palese C, Al-Kawas FH. Repeat intentional foreign body ingestion: the importance of a multidisciplinary approach. Gastroenterology \& hepatology. 2012;8(7):485-6. 3. Volpi A Fau - Laforgia R, Laforgia R Fau -

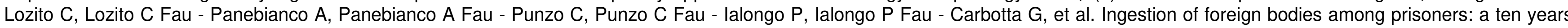

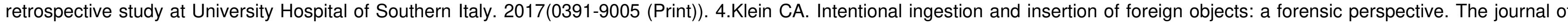

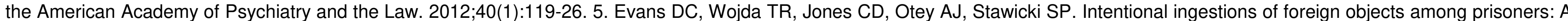

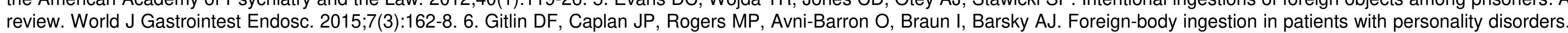

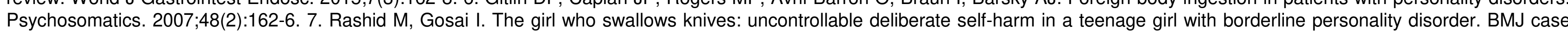
reports. $2011 ; 2011$ 